\title{
https://doi.org/10.1007/s00350-021-6059-8
}

\section{Risiko- und Qualitätsmanagement im Gesundheitswesen}

\author{
Entwicklung und Tendenzen 2020/2021
}

Karl Otto Bergmann und Carolin Wever

\section{Einleitung}

Drei Hauptthemen beherrschen die gegenwärtige wissenschaftliche, aber auch politische Diskussion über die Zukunft des Gesundheitswesens: die Digitalisierung der Krankenhäuser unter Wahrung der Informationssicherheit, die Auswirkungen der Corona-Pandemie und schließlich die Hochwasserschäden und Hochwassergefahren sowie die vorhandenen Risikomanagementsysteme hinsichtlich des Schutzes vor solchen Naturkatastrophen. Diese Themen prägen auch unseren Jahresbericht 2020/2021 ${ }^{1}$, wobei auch weitere wichtige Facetten des Risiko- und Qualitätsmanagements nicht vernachlässigt werden sollen, so die Verbindung von Entlass- und Belegungsmanagement, die Qualitätssicherung in Sepsisfällen sowie das Management im Krankenhaus zur Sicherung der Personenidentität.

Risiko- und Qualitätsmanagement gewinnen insbesondere in der Krankenhauslandschaft immer größere Bedeutung, so dass wir nur die wichtigsten Themen aufgreifen und skizzieren können. Der Leser sei deshalb ausdrücklich darauf hingewiesen, dass soeben in 3. Auflage das umfassende Handbuch „Qualitätsmanagement im Krankenhaus“ von Heidemarie Haeske-Seeberg erschienen ist, das auf über 600 S. nicht nur die geschichtliche Entwicklung von Qualitätsmanagement und Qualitätssicherung, die gesetzlichen Grundlagen und die Instrumente der gesetzlichen Qualitätssicherung sowie die vom G-BA vorgegebenen Instrumente des Qualitätsmanagements darstellt, sondern auch mit vielen praktischen Beispielen Qualitätsziele, Leitbild, Qualitätsmessung, Patientenbefragung, Benchmarking und externe Qualitätsvergleiche, Audits und weitere Bereiche aufzeigt.

Welche Bedeutung insbesondere die Digitalisierung und die IT-Sicherheit in Kliniken hat, ergibt sich aus der Tatsache, dass Bundestag und Bundesrat im Herbst 2020 das Krankenhauszukunftsgesetz verabschiedet haben und ein Milliardenbetrag zur Verfügung gestellt wird, um Digitalisierung einerseits und IT-Sicherheit andererseits im deutschen Gesundheitswesen fest zu etablieren.

\section{BSI-Studie zur IT-Sicherheit}

Sehr viele Krankenhäuser in Deutschland haben sich bisher nicht vor Cyberangriffen geschützt. Die bekannt gewordenen Angriffe, beispielsweise auf das Universitätsklinikum Düsseldorf ${ }^{2}$, haben das Bundesamt für Sicherheit in der Informationstechnik (BSI) im Juni 2021 veranlasst, eine Studie zur Informationssicherheit in der stationären medizinischen Versorgung zu veröffentlichen ${ }^{3}$. Die Studie bestätigt, dass die rund 1800 öffentlichen, freien gemeinnützigen und privaten Krankenhäuser, die nicht unter die KRITIS-Regulierung fallen, keine IT-spezifische Sicherheitskultur haben. Es sind ungenügend finanzielle Mittel für Investitionen in Hard- und Software, die Durchführung von Schulungen wie für den personellen Aufwand zur Dokumentation, Uberprüfung, Auditierung und kontinu-

Rechtsanwalt Prof. Dr. iur. Karl Otto Bergmann

und Rechtsanwältin Dr. iur. Carolin Wever,

Kanzlei Bergmann und Partner,

Josef-Schlichter-Allee 38, 59063 Hamm, Deutschland ierlichen Weiterentwicklung der Compliance-Regelungen vorhanden. Zur Absicherung der Systeme und der Prozesse ist nach Auffassung des BSI vor allem ein Management erforderlich, welches die Sicherheit des Zugangs zu Daten von IT-Anwendungen oder medizinischen Geräten gewährleistet. Beklagt wird, dass häufig wechselndes Personal auf Grund von Schichtwechsel, Teilzeitarbeit, Leiharbeit und Belegärzte die Sensibilisierung der Beschäftigten im Bereich IT-Sicherheit verhindert. Netztrennung und Netzsegmentierung im Medizintechnik-Netz, Verwaltungsnetz, Applikationsnetz und Patienten-/Gästenetz ist oftmals nicht vollständig vollzogen. Weitere Einschränkungen ergeben sich nach Auffassung des BSI auch durch Restriktionen der Speichersysteme. Wegen immer stärker zunehmender Datenmengen kommt es schon jetzt zu Kapazitätsengpässen, dieser Trend wird zunehmen.

\section{Das Krankenhauszukunftsgesetz}

Am 9.10.2020 hat der Bundesrat das Krankenhauszukunftsgesetz (Gesetz für ein Zukunftsprogramm für Krankenhäuser, verabschiedet durch den Bundestag am 18.9.2020) gebilligt ${ }^{4}$. Das Krankenhauszukunftsgesetz hat nach dem Willen der Politik das Ziel, die Weichen im deutschen Krankenhauswesen in Richtung Digitalisierung zu stellen und die Krankenhäuser zu unterstützen, in moderne Notfallkapazitäten, die Digitalisierung und IT-Sicherheit investieren zu können. Der Bundeshaushalt stellt hierfür 3 Milliarden Euro, die Länder weitere 1,3 Milliarden Euro zur Verfügung, sodass insgesamt 4,3 Milliarden Euro im Haushalt angesetzt sind. Der KrankenhauszukunftsFond wird beim Bundesamt für Soziale Sicherung (BAS) mit 3 Milliarden Euro aus der Liquiditätsreserve des Gesundheitsfonds errichtet. Die Förderung dient der eigentlichen Patientenbehandlung, insbesondere der Digitalisierung und Modernisierung von Begleitleistungen. Hierzu gehören beispielsweise die Ablauforganisation, die Dokumentation und Kommunikation, die technische Verbesserung von Telemedizin, Robotik und Hightech-Medizin.

Mindestens $15 \%$ der Fördermittel sind in die Informationssicherheit zu investieren. In $\$ 19$ dieses Gesetzes sind die förderungsfähigen Vorhaben im Einzelnen aufgeführt. Erwähnt seien an dieser Stelle nur die Anpassung der technischen Ausstattung von Notaufnahmen und die Einrichtung von Patientenportalen zum digitalen Informationsaustausch sowie die elektronische Dokumentation von Pflege- und Behandlungsleistungen. Allerdings müssen Projekte und Anschaffungen, die im Rahmen des Krankenhauszukunftsfonds finanziert werden können, später aus eigenen Mitteln betrieben und gewartet werden. Deshalb ist eine sorgfältige Planung mit Berücksichtigung der gesamten Lebenszykluskosten eines solchen Vorhabens unerlässlich.

1) Der Beitrag schließt an MedR 2020, 917 ff. an

2) Vgl. nur etwa https://www.uniklinik-duesseldorf.de/ueber-uns/ pressemitteilungen/detail/it-ausfall-an-der-uniklinik-duesseldorf, zuletzt abgerufen am 28.9.2021.

3) https://www.bsi.bund.de/Shared-Docs/Downloads/DE/BSI/ Publikationen/Studien/KRITIS/Studie Informationssicherheit_stationaere_med_Versorgung.pdf?_blob=publicationFile\& $\mathrm{v}=3$, zuletzt abgerufen am 28.9.2021.

4) BGBl. I S. 2208. 


\section{CIRS-NRW - Bericht des II. Quartals 2021 - „Achtsamkeit ist gefragt!“‘}

Bereits in unseren früheren Jahresberichten haben wir auf die Notwendigkeit hingewiesen, dass digitale Prozesse im Krankenhaus gerade in der Einführungsphase nicht nur die Chance versprechen, eine Optimierung klinisch interner Prozesse, eine Entlastung des medizinischen und administrativen Personals und eine höhere Patientensicherheit zu gewährleisten, sondern auch Gefahren für die Behandlungsprozesse im Krankenhaus bringen. Neben unseren Berichten sei auf die Berichte von CIRS-NRW mit den Ziffern 215529, 217596, 218238 und 218297 verwiesen ${ }^{5}$. CIRS-NRW weist zu Recht auf die Gefahren unsicherer IT-Infrastrukturen, unsauber programmierter digitaler Patientenakten und auf die Gefahr von Medienbrüchen beim Übergang vom analogen auf digitale Prozessschritte hin. CIRS-NRW betont, dass sich gerade in der Einführungsphase digitaler Technologien in Krankenhäusern die Behandlungs- und Therapieprozesse ständig ändern. Gefahren, die von Medienbrüchen zwischen analoger und digitaler Dokumentation ausgehen, sind besonders hoch. Daher ist nach Auffassung von CIRS-NRW eine stetige professionelle Risiko- und Gefahrenanalyse der sich verändernden Behandlungs- und Therapieprozesse und eine zeitnahe Fehlerbehebung zur Gewährleistung der Patientensicherheit dringend geboten.

Neben dem Thema Digitalisierung der Krankenhäuser steht, wie bereits betont, auch das Thema Corona-Pandemie im Fokus von Risiko- und Qualitätsmanagement. In der Diskussion sind insbesondere die Leitlinien der medizinischen Fachgesellschaften und die möglichst qualitätsvolle und risikofreie Bewältigung der Infektionswellen.

\section{S3-Leitlinie COVID 19}

Die bisherigen Empfehlungen zur stationären Therapie von Patientinnen und Patienten mit COVID-19 sind durch 14 verschiedene medizinische Fachgesellschaften überarbeitet worden $^{6}$. In Zusammenarbeit mit dem COVID-19-Evidenzökosystem-Projekt (CEOsys) wurden entscheidende therapeutisch relevante Fragestellungen mit systematischen Evidenzthesen hinterlegt. Notwendig ist eine fächerübergreifende Betrachtung, wie der Koodinator der Leitlinie, Prof. Dr. Kluge, darlegte: ,Derzeit werden täglich hunderte neue wissenschaftliche Arbeiten zu COVID-19 publiziert. Die vorliegende Leitlinie bezieht alle neuen und gesicherten Erkenntnisse mit ein und ist für ein strukturiertes, sicheres und ressourcenschonendes Management und COVID19-Patienten im Krankenhaus unerlässlich"7. Hinsichtlich der medikamentösen Behandlung gilt weiterhin, dass eine klinische Wirksamkeit medikamentöser Therapie bei moderater bis schwerer COVID-19-Erkrankung hospitalisierter Patienten nur für Dexamethason mit ausreichender Sicherheit nachgewiesen ist. Andere Medikamente können mangels fehlender Evidenz noch nicht regelhaft zum Einsatz empfohlen werden. Die S3-Leitlinie ist abrufbar auf der Website der AWMF.

\section{CIRS-NRW Bericht des II. Quartals 2020}

CIRS-NRW weist darauf hin, dass gerade bei disruptiven Entwicklungen der Pandemie, die ein spontanes, intuitives Vorgehen und schnelle Entscheidungen erfordern, die Gefahr von kritischen Ereignissen sehr groß ist ${ }^{8}$. Als Maßnahme zur Bewältigung solcher Krisen empfiehlt sich die Aufstellung eines Covid-Auditplans, Prüfung und Umsetzung der beschlossenen Maßnahmen durch Begehung, Bereitstellung von Covid-19-Dokumenten im Dokumentenlenkungssystem, Erstellung eines Covid-19-Newsletters, Erstellung eines Algorithmus und von Dokumentationshil- fen zur Triage, Erstellung von Flussdiagrammen und SOPs $\mathrm{zu}$ intensivmedizinischen Behandlungen, Assessments zur Bauchlagerung von Covid-19-Patienten, Erstellung von Filmen zum Einsatz und Wechsel von Schutzkleidung. CIRS-NRW weist aber zu Recht drauf hin, dass bisher die Kompetenz von Qualitäts- und Risikomanagerinnen und -managern bei der Risikoidentifizierung und -bewältigung wenig einbezogen wurde. Agiles Qualitäts- und Risikomanagement wird aber nach Auffassung von CIRS-NRW nach der Corona-Krise stärker gefragt sein. Reorganisationen und anstehende Reformen im Gesundheitswesen werden weitreichende Einflüsse auf Strukturen und Prozesse im Gesundheitswesen haben. Wir verweisen in diesem Zusammenhang auf die Untersuchungen einer Expertenkommission zur Klärung des schweren Corona-Ausbruchs im Bergmannklinikum Potsdam im März/April $2020^{9}$. Die Expertenkommission hat ermittelt, dass das Klinikum grundlegende Defizite in Hygiene und Organisation der Klinik gehabt hat, die Klinikleitung hat Versäumnisse im Umgang mit dem Ausbruch des Virus eingestanden, der Ausbruch wurde zu spät erkannt und deshalb wurde nicht adäquat reagiert. Es kann nicht ausgeschlossen werden, dass grundlegende organisatorische und hygienische Defizite, Fehleinschätzungen und Fehlhandlungen beim Ausbruchsgeschehen und Mängel der internen Zusammenarbeit bei früherem Qualitäts- und Risikomanagement vermieden worden wären.

\section{Hochwasserschäden und Risikomanagement}

Ein weiteres zentrales Thema für Krankenhäuser war das Risikomanagement zur Vermeidung von Hochwasserschäden, wie sie im Norden von Rheinland/Pfalz bzw. im Süden von NRW eingetreten sind. Bereits früher waren Hochwasserschäden Anlass für ein Risikomanagement ${ }^{10}$. Ebenso ergab sich hieraus die Notwendigkeit, die vorhandenen Risikomanagementsysteme hinsichtlich des Schutzes vor solchen Naturkatastrophen zu überprüfen. So wurden beispielsweise im August 2021 im Rahmen einer Risikomanagementübung am Klinikum Bitterfeld der Aufbau einer mobilen Hochwasserschutzanlage von $500 \mathrm{~m}$ Länge als Übung durchgeführt ${ }^{11}$. Alle technischen Mitarbeiter müssen in der Lage sein, im Ernstfall die Hochwasserschutzwand so schnell wie möglich aufzubauen. Anlässlich der Jahrhundertflut im August 2002 zerstörte die Flut das Herzstück des Gesundheitszentrums mit allen technischen Anlagen und Versorgungseinrichtungen. Die regelmäßige Kontrolle der Funktionstüchtigkeit des Hochwasserschutzes bedarf eines systematischen Risikomanagements.

\section{Risikomanagement zur Vermeidung von Patientenverwechslung}

Das CIRS-Team des BÄK wies in einem Bericht darauf hin, dass Patientenverwechslungen auf Grund einer $\mathrm{Na}$ -

5) Abzurufen unter https://www.cirsmedical.ch/DeutschlandPlus/ m_files/cirs.php?seitennr=AEZQ, zuletzt abgerufen am 28.9.2021.

6) AWMF-Register-Nr. 113/001, abzurufen unter https://www. awmf.org, zuletzt abgerufen am 28.9.2021.

7) https://idw-online.de/de/news?print=1\&id=763654; zuletzt abgerufen am 28.9.2021.

8) https://www.cirsmedical.de/nrw/pdf/bericht-quartal-20202.pdf; zuletzt abgerufen am 28.9.2021.

9) https://www.pnn.de/downloads/26832858/4/abschlussbericht.pdf, zuletzt abgerufen am 28.9.2021.

10) Vgl. dazu nur etwa https://www.bibliomedmanager.de/fw/artikel/35715-wie-sich-kliniken-gegen-die-flut-wappnen, zuletzt abgerufen am 28.9.2021.

11) https://www.mz.de/lokal/bitterfeld/hochwasserschutz-das-bitterfelder-klinikum-ubt-fur-den-ernstfall-3237697, zuletzt abgerufen am 28.9.2021. 
mensähnlichkeit bei der Medikamentenausgabe immer wieder vorkommen, insbesondere bei erhöhten Arbeitsaufkommen ${ }^{12}$. Patientenverwechslungen egal welcher Art und in welchem Zusammenhang können fatale Folgen nach sich ziehen. Deshalb muss bei Namensähnlichkeit bzw. -gleichheit von Patienten ein Hinweis z. B. in der Anmeldeliste/KISS hinterlegt werden. Auch sollte eine aktive Patientenidentifikation vor jeder Untersuchung oder Medikamentenabgabe erfolgen. Die zuständigen Mitarbeiter sind auf die Notwendigkeit der korrekten Patientenidentifikation hinzuweisen und zu schulen. Eine wichtige Sicherheitsbarriere ist die 6-R-Regel (richtige Person, richtiges Arzneimittel, richtige Dosierung/Konzentration, richtiger Applikationsweg, richtiger Zeitpunkt, richtige Dokumentation). Verwiesen sei auch auf die Handlungsempfehlung der KVWL Westfalen-Lippe „Sichere Patientenidentifikation in der ambulanten ärztlichen Versorgung“"13.

\section{Kampagne „,Deutschland erkennt Sepsis““}

Das Aktionsbündnis Patientensicherheit e.V. (APS), die Sepsisstiftung, die Deutsche Sepsis-Hilfe, die Universitätsmedizin Greifswald und der Verband der Ersatzkassen (VDEK) haben eine Kampagne ,Deutschland erkennt Sepsis“ ins Leben gerufen. Jedes Jahr sterben mehr als 75.000 Menschen in Deutschland an einer Sepsis. Rund 20.000 Todesfälle jährlich sowie viele Spätfolgen wie Amputationen und Depressionen gelten als vermeidbar. Die Krankenhaussterblichkeit bei schwerer Sepsis liegt in Deutschland bei $42 \%$ im Vergleich zu vielen anderen Ländern. Dies ist deutlich erhöht. Für die USA werden 23\%, für England $32 \%$ und für Australien $18 \%$ angegeben ${ }^{14}$. Die Kampagne soll das Krankenhauspersonal sensibilisieren und stellt umfangreiche Informationsmaterialien für medizinisches Fachpersonal zur Verfügung ${ }^{15}$. Patientensicherheit als eigenes Lernfeld mit allen dazugehörigen Kenntnissen und Fertigkeiten gehört an zentraler Stelle in die Aus- und Weiterbildung aller Gesundheitsberufe. Der Verdacht auf Sepsis erfordert eine schnelle und zuverlässige Blutkulturdiagnostik. Gefordert wird deshalb als klinischer Standard eine rund um die Uhr verfügbare Blutkulturdiagnostik, deren Abläufe auf den zeitkritischen Ablauf einer Sepsis abgestimmt sein müssen.

\section{Qualitätssicherung in Sepsisfällen durch G-BA}

Im Hinblick auf die deutlich zu hohe Zahl von Sepsisfällen in Deutschland hat die Patientenvertretung beim G-BA schon 2018 einen Antrag auf Entwicklung einer Qualitätssicherungsmaßnahme zur Verbesserung der Erkenntnis und Behandlung von Sepsis gestellt. Das daraufhin beauftragte Institut für Qualität und Transparenz im Gesundheitswesen (IQTIG) ist mit der Entwicklung eines Qualitätssicherungsverfahrens in Krankenhäusern sowie Notaufnahmen und Rettungsstellen beauftragt und wird zum 30.1.2022 dem G-BA einen Abschlussbericht übermitteln ${ }^{16}$. Zusätzlich hat das Aktionsbündnis Patientensicherheit im Januar 2021 eine Handlungsempfehlung mit dem Titel „Sepsis geht alle an - Handlungsempfehlungen für das Management und Gesundheitswesen " veröffentlicht ${ }^{17}$. Weitere Hinweise auch zu bereits durchgeführten Klinikprojekten bietet der bereits oben zitierte Fachbeitrag von Fechner und Dreyer ${ }^{18}$.

\section{Verbindung von Entlass- und Belegungsmanagement}

Die Schnittstelle an der Sektorengrenze zwischen Akutund Rehabilitationsmedizin, also die Überweisung des Patienten aus dem Krankenhaus in die Reha-Klinik, bietet im Hinblick auf die notwendige Kommunikation und Dokumentation der ärztlichen und pflegerischen Maßnahmen in der Praxis immer Schwierigkeiten und damit Haftungsgefahren. Die Unternehmen Asklepios und Median wollen deshalb innovative Wege gehen. Ein neuer technischer sogenannter „Brückenschlag“ (care-bridge) verbindet das digitale Entlassmanagement der Asklepios Krankenhäuser über ein zentrales Reservierungsservice (ZAS) mit den Median-Kliniken ${ }^{19}$. Die digitale Verknüpfung ist aus Datenschutzperspektive durch eine verschlüsselte Ende-zuEnde Datenübertragung sicherer als das herkömmliche Fax oder eine E-Mail. Damit wird der Prozess der Suche und der Abwicklung einer Verlegung gleichzeitig erleichtert. Gleichzeitig bedeutet dies für die Akutklinik eine zeitgerechtere Abverlegung.

\section{Zusammenfassung und Ausblick}

Unser ausschnittartiger Überblick über Tendenzen und Entwicklung von Qualitäts- und Risikomanagement zeigt vielfältige Zukunftsaufgaben aller im Gesundheitsbereich Tätigen auf.

In Anbetracht der vielfältigen Aufgaben für die Zukunft, die in einem engen Zusammenwirken vorbereitet werden müssen, sei abschließend an die Worte des griechischen Staatsmannes Perikles erinnert: „Es kommt nicht darauf an, die Zukunft vorauszusagen, sondern darauf, auf die Zukunft vorbereitet zu sein."

12) Fall-Nr: 226446, abzurufen unter https://www.cirsmedical.ch/ DeutschlandPlus/m_files/cirs.php?seitennr=AEZQ, zuletzt abgerufen am 28.9.2021.

13) https://www.kvwl.de/arzt/qsqm/patientensicherheit/handl_ empf_patientenidentifikation.pdf, zuletzt abgerufen am 28.9. 2021.

14) Fechner/Dreyer, Das Krankenhaus 2021, 416.

15) Abzurufen unter https://www.deutschland-erkennt-sepsis.de/ materialien-fuer-medizinischespersonal, zuletzt abgerufen am 28.9.2021.

16) https://patientenvertretung.g-ba.de/neuigkeiten/qualitaetssicherung/sepsis_-g-ba-beauftragt-iqtig-mit-entwicklung-einesqs-verfahrens/, zuletzt abgerufen am 28.9.2021.

17) https://www.aps-ev.de/wp-content/uploads/2021/02/APSHE_Sepsis_Management.pdf, zuletzt abgerufen am 28.9.2021.

18) Fechner/Dreyer, Das Krankenhaus 2021, 416.

19) https://www.kma-online.de/aktuelles/it-digital-health/detail/ asklepios-und-median-koppeln-entlass-und-belegungsmanagement-a-44037, zuletzt abgerufen am 28.9.2021. 